\title{
NEW GRONWALL-OU-IANG TYPE INTEGRAL INEQUALITIES AND THEIR APPLICATIONS
}

\author{
YEOL JE CHO ${ }^{1}$, YOUNG-HO KIM $^{\bowtie 2}$ and JOSIP PEČARIĆ ${ }^{3}$
}

(Received 17 July, 2007; revised 11 December, 2008)

\begin{abstract}
Some new Gronwall-Ou-Iang type integral inequalities in two independent variables are established. We also present some of its application to the study of certain classes of integral and differential equations.

2000 Mathematics subject classification: primary 26D10; secondary 26D15, 34K10, $35 \mathrm{~B} 35$.

Keywords and phrases: integral inequalities, retarded integral inequality, retarded differential equations, partial delay differential equations.
\end{abstract}

\section{Introduction}

One of the most useful methods available for studying a linear and nonlinear system of ordinary differential equations is the use of linear and nonlinear integral inequalities which provide explicit bounds on the unknown functions. Over the last scores of years several new linear and nonlinear integral inequalities have been developed in order to study the behaviour of solutions of such systems. See, for example, [1-13].

In the study of the boundedness of solutions to linear second-order differential equations, Ou-Iang [9] established and applied the following useful nonlinear integral inequality. If $u, f$ are nonnegative continuous functions on $R_{+}=[0, \infty), u_{0} \geq 0$ is a constant and

$$
u^{2}(t) \leq u_{0}^{2}+2 \int_{0}^{t} f(s) u(s) d s
$$

\footnotetext{
${ }^{1}$ Department of Mathematics Education, The Research Institute of Natural Sciences, College of Education, Gyeongsang National University, Chinju 660-701, Republic of Korea; e-mail: yjcho@gnu.ac.kr.

${ }^{2}$ Department of Applied Mathematics, Changwon National University, Changwon, Kyung-Nam 641-773, Republic of Korea; e-mail: yhkim@sarim.changwon.ac.kr.

${ }^{3}$ Faculty of Textile Technology, University of Zagreb, Pierottijeva 6, 1000 Zagreb, Croatia; e-mail: pecaric@element.hr.

(c) Australian Mathematical Society 2009, Serial-fee code 1446-1811/09 \$16.00
} 
for all $t \in R_{+}$, then

$$
u(t) \leq u_{0}+\int_{0}^{t} f(s) d s, \quad t \in R_{+} .
$$

Like the Gronwall type inequalities, (1.1) is also used to obtain the boundedness of solutions to the unknown function. Furthermore, this result has been extended and generalized by many authors (see [1-3, 5-7, 11-13]). Therefore, the integral inequalities of this type are usually known as the Gronwall-Ou-Iang type inequalities.

Recently, Pachpatte in [13] obtained a useful upper bound involving functions in two independent variables on the inequality

$$
u^{p}(x, y) \leq c+p \sum_{i=1}^{n}\left[\int_{\alpha_{i}\left(x_{0}\right)}^{\alpha_{i}(x)} \int_{\beta_{i}\left(x_{0}\right)}^{\beta_{i}(y)}\left[a_{i}(s, t) u^{p}(s, t)+b_{i}(s, t) u(s, t)\right] d t d s\right]
$$

and its variants, under some suitable conditions on the functions involved in (1.2) and including the constant $p>1$. These inequalities are applied to study the boundedness of the solutions of the retarded partial differential equation (1.3) with the initial boundary conditions (1.4)

$$
\begin{aligned}
& \frac{\partial}{\partial y}\left[z^{p-1}(x, y) \frac{\partial}{\partial x} z(x, y)\right] \\
& \quad=F\left[x, y, z\left(x-h_{1}(x), y-g_{1}(y)\right), \ldots, z\left(x-h_{n}(x), y-g_{n}(y)\right)\right], \\
& z\left(x, y_{0}\right)=e_{1}(x), \quad z\left(x_{0}, y\right)=e_{2}(y), \quad e_{1}\left(x_{0}\right)=e_{2}\left(y_{0}\right)=0 .
\end{aligned}
$$

The authors Cheung [2], Cheung-Ma [3], Dragomir-Kim [5, 6] and Pachpatte [13] established additional new Gronwall-Ou-Iang type integral inequalities involving functions of two independent variables. Our main aim here, motivated by the works of Cheung, Cheung-Ma, Dragomir-Kim and Pachpatte, is to establish some new and more general Gronwall-Ou-Iang type integral inequalities with two independent variables which are useful in the analysis of certain classes of partial differential equations.

\section{Main results}

We shall introduce some notation. Let $R$ denote the set of real numbers and $R_{+}$ $=[0, \infty), I=\left[t_{0}, T\right)$ be the given subsets of $R$. Let $\Delta=I_{1} \times I_{2}$, where $I_{1}=\left[x_{0}, X\right)$ and $I_{2}=\left[y_{0}, Y\right)$ are the given subsets of real numbers $R$. Denote by $C^{i}(M, N)$ the class of all $i$-times continuously differentiable functions defined on set $M$ to the set $N$. The first-order partial derivatives of a function $z(x, y)$ defined for $x, y \in R$ with respect to $x$ and $y$ are denoted by $D_{1} z(x, y)$ and $D_{2} z(x, y)$, respectively.

LEMMA 2.1. Let $u, a_{i} \in C\left(\triangle, R_{+}\right), \alpha_{i} \in C^{1}\left(I_{1}, I_{1}\right), \beta_{i} \in C^{1}\left(I_{2}, I_{2}\right)$ be nondecreasing with $\alpha_{i}(x) \leq x$ on $I_{1}, \beta_{i}(y) \leq y$ on $I_{2}$ for $i=1,2, \ldots, n$. Let $\varphi \in C\left(R_{+}, R_{+}\right)$be an increasing function with $\varphi(\infty)=\infty$ and c be a nonnegative constant. Moreover, 
let $w_{1} \in C\left(R_{+}, R_{+}\right)$be a nondecreasing function with $w_{1}>0$ on $(0, \infty)$. If

$$
\varphi(u(x, y)) \leq c+\sum_{i=1}^{n}\left[\int_{\alpha_{i}\left(x_{0}\right)}^{\alpha_{i}(x)} \int_{\beta_{i}\left(x_{0}\right)}^{\beta_{i}(y)} a_{i}(s, t) w_{1}(u(s, t)) d t d s\right]
$$

for all $x \in I_{1}, y \in I_{2}$, then, for $x_{0} \leq x \leq x_{1}, y_{0} \leq y \leq y_{1}$ with $x_{1} \in I_{1}, y_{1} \in I_{2}$,

$$
\begin{array}{r}
u(x, y) \leq \varphi^{-1}\left\{G^{-1}\left[G(c)+\sum_{i=1}^{n}\left(\int_{\alpha_{i}\left(x_{0}\right)}^{\alpha_{i}(x)} \int_{\beta_{i}\left(x_{0}\right)}^{\beta_{i}(y)} a_{i}(s, t) d t d s\right)\right]\right\} \\
\text { where } \quad G(r)=\int_{r_{0}}^{z} \frac{d s}{w_{1}\left[\varphi^{-1}(s)\right]}, \quad r \geq r_{0}>0,
\end{array}
$$

$\varphi^{-1}, G^{-1}$ are, respectively, the inverse of $\varphi, G$ and $x_{1} \in I_{1}, y_{1} \in I_{2}$ are chosen so that

$$
\begin{gathered}
G(c)+\sum_{i=1}^{n}\left(\int_{\alpha_{i}\left(x_{0}\right)}^{\alpha_{i}(x)} \int_{\beta_{i}\left(x_{0}\right)}^{\beta_{i}(y)} a_{i}(s, t) d t d s\right) \in \operatorname{Dom}\left(G^{-1}\right), \\
G^{-1}\left[G(c)+\sum_{i=1}^{n}\left(\int_{\alpha_{i}\left(x_{0}\right)}^{\alpha_{i}(x)} \int_{\beta_{i}\left(x_{0}\right)}^{\beta_{i}(y)} a_{i}(s, t) d t d s\right)\right] \in \operatorname{Dom}\left(\varphi^{-1}\right)
\end{gathered}
$$

for all $x \in\left[x_{0}, x_{1}\right]$ and $y \in\left[y_{0}, y_{1}\right]$.

PROOF. Define a positive function $z(x, y)$ by

$$
z(x, y)=c+\epsilon+\sum_{i=1}^{n}\left[\int_{\alpha_{i}\left(x_{0}\right)}^{\alpha_{i}(x)} \int_{\beta_{i}\left(y_{0}\right)}^{\beta_{i}(y)} a_{i}(s, t) w_{1}(u(s, t)) d t d s\right],
$$

where $\epsilon$ is an arbitrary small positive number. Then (2.1) can be restated as

$$
u(x, y) \leq \varphi^{-1}[z(x, y)] .
$$

It is easy to observe that $z(x, y)$ is a continuous nondecreasing function for all $x \in I_{1}, y \in I_{2}$ and

$$
\begin{aligned}
D_{1} z(x, y) & =\sum_{i=1}^{n}\left[\int_{\beta_{i}\left(y_{0}\right)}^{\beta_{i}(y)} a_{i}\left(\alpha_{i}(x), t\right) w_{1}\left(u\left(\alpha_{i}(x), t\right)\right) d t\right] \alpha_{i}^{\prime}(x) \\
& \leq \sum_{i=1}^{n}\left[\int_{\beta_{i}\left(y_{0}\right)}^{\beta_{i}(y)} a_{i}\left(\alpha_{i}(x), t\right) w_{1}\left(\varphi^{-1}\left[z\left(\alpha_{i}(x), t\right)\right]\right) d t\right] \alpha_{i}^{\prime}(x) \\
& \leq w_{1}\left(\varphi^{-1}[z(x, y)]\right) \sum_{i=1}^{n}\left[\int_{\beta_{i}\left(y_{0}\right)}^{\beta_{i}(y)} a_{i}\left(\alpha_{i}(x), t\right) d t\right] \alpha_{i}^{\prime}(x) .
\end{aligned}
$$

Using the monotonicity of $\varphi^{-1}$ and $w_{1}$, we deduce

$$
w_{1}\left(\varphi^{-1}[z(x, y)]\right) \geq w_{1}\left(\varphi^{-1}\left[z\left(x_{0}, y_{0}\right)\right]\right)=w_{1}\left(\varphi^{-1}[c+\epsilon]\right)>0 .
$$


From the definition of $G$, the inequalities (2.4) and (2.5) give

$$
D_{1} G(z(x, y))=\frac{D_{1} z(x, y)}{w_{1}\left(\varphi^{-1}[z(x, y)]\right)} \leq \sum_{i=1}^{n}\left[\int_{\beta_{i}\left(y_{0}\right)}^{\beta_{i}(y)} a_{i}\left(\alpha_{i}(x), t\right) d t\right] \alpha_{i}^{\prime}(t)
$$

Keeping $y$ fixed in (2.6), setting $x=\sigma$ and integrating it with respect to $\sigma$ from $x_{0}$ to $x, x \in I_{1}$ and making the change of variable, we obtain

$$
G(z(x, y)) \leq G\left(z\left(x_{0}, y\right)\right)+\sum_{i=1}^{n} \int_{\alpha_{i}\left(x_{0}\right)}^{\alpha_{i}(x)} \int_{\beta_{i}\left(y_{0}\right)}^{\beta_{i}(y)} a_{i}(s, t) d t d s .
$$

Since $G^{-1}(z)$ is increasing, letting $\epsilon \rightarrow 0$ yields

$$
z(x, y) \leq G^{-1}\left[G(c)+\sum_{i=1}^{n} \int_{\alpha_{i}\left(x_{0}\right)}^{\alpha_{i}(x)} \int_{\beta_{i}\left(y_{0}\right)}^{\beta_{i}(y)} a_{i}(s, t) d t d s\right]
$$

for

$$
G(c)+\sum_{i=1}^{n} \int_{\alpha_{i}\left(x_{0}\right)}^{\alpha_{i}(x)} \int_{\beta_{i}\left(y_{0}\right)}^{\beta_{i}(y)} a_{i}(s, t) d t d s \in \operatorname{Dom}\left(G^{-1}\right) .
$$

Using (2.7) in (2.3), we get the required inequality in (2.2). This completes the proof of the lemma.

In what follows, for any functions $f_{i}, g_{i} \in C\left(R_{+}, R_{+}\right)$, define

$$
I_{\alpha_{i}, \beta_{i}}\left[f_{i}(s, t)+g_{i}(s, t)\right] \equiv \int_{\alpha_{i}\left(x_{0}\right)}^{\alpha_{i}(x)} \int_{\beta_{i}\left(y_{0}\right)}^{\beta_{i}(y)}\left[f_{i}(s, t)+g_{i}(s, t)\right] d t d s .
$$

Theorem 2.2. Let $u, f_{i} \in C\left(\triangle, R_{+}\right), \alpha_{i} \in C^{1}\left(I_{1}, I_{1}\right), \beta_{i} \in C^{1}\left(I_{2}, I_{2}\right)$ be nondecreasing with $\alpha_{i}(x) \leq x$ on $I_{1}, \beta_{i}(y) \leq y$ on $I_{2}$ for $i=1,2, \ldots, n$. Let $c$ be $a$ nonnegative constant. Moreover, assume that $\varphi \in C\left(R_{+}, R_{+}\right)$and $w_{1} \in C\left(R_{+}, R_{+}\right)$ are defined as in Lemma 2.1. If

$$
\varphi(u(x, y)) \leq c+\sum_{i=1}^{n} I_{\alpha_{i}, \beta_{i}}\left[f_{i}(s, t) u(s, t) w_{1}(u(s, t))\right]
$$

for all $x \in I_{1}, y \in I_{2}$, then, for $x_{0} \leq x \leq x_{2}, y_{0} \leq y \leq y_{2}$ with $x_{2} \in I_{1}, y_{2} \in I_{2}$,

$$
u(x, y) \leq \varphi^{-1}\left\{\Omega^{-1}\left[G^{-1}\left(G[\Omega(c)]+\sum_{i=1}^{n} I_{\alpha_{i}, \beta_{i}}\left[f_{i}(s, t)\right]\right)\right]\right\}
$$

where

$$
\Omega(r)=\int_{r_{0}}^{z} \frac{d s}{\varphi^{-1}(s)}, \quad r \geq r_{0}>0, \quad G(z)=\int_{z_{0}}^{z} \frac{d s}{w_{1}\left[\varphi^{-1}\left(\Omega^{-1}(s)\right)\right]}, \quad z \geq z_{0}>0,
$$


$\Omega^{-1}, \varphi^{-1}, G^{-1}$ are, respectively, the inverses of $\Omega, \varphi, G$ and $x_{2} \in I_{1}, y_{2} \in I_{2}$ are chosen so that

$$
\begin{gathered}
G(\Omega(c))+\sum_{i=1}^{n} I_{\alpha_{i}, \beta_{i}}\left[f_{i}(s, t)\right] \in \operatorname{Dom}\left(G^{-1}\right), \\
G^{-1}\left[G(\Omega(c))+\sum_{i=1}^{n} I_{\alpha_{i}, \beta_{i}}\left[f_{i}(s, t)\right]\right] \in \operatorname{Dom}\left(\Omega^{-1}\right), \\
\Omega^{-1}\left\{G^{-1}\left[G(\Omega(c))+\sum_{i=1}^{n} I_{\alpha_{i}, \beta_{i}}\left[f_{i}(s, t)\right]\right]\right\} \in \operatorname{Dom}\left(\varphi^{-1}\right)
\end{gathered}
$$

for all $x \in\left[x_{0}, x_{2}\right]$ and $y \in\left[y_{0}, y_{2}\right]$.

PROOF. Let us first assume that $c>0$. Define a positive function $z(x, y)$ by

$$
z(x, y)=c+\sum_{i=1}^{n} I_{\alpha_{i}, \beta_{i}}\left[f_{i}(s, t) u(s, t) w_{1}(u(s, t))\right] .
$$

Then $z(x, y)>0, z\left(x_{0}, y\right)=z\left(x, y_{0}\right)=c$ and (2.8) can be restated as

$$
u(x, y) \leq \varphi^{-1}[z(x, y)] .
$$

It is easy to observe that $z(x, y)$ is a continuous nondecreasing function for all $x \in I_{1}, y \in I_{2}$ and

$$
\begin{aligned}
D_{1} z(x, y) & =\sum_{i=1}^{n}\left[\int_{\beta_{i}\left(y_{0}\right)}^{\beta_{i}(y)}\left[f_{i}\left(\alpha_{i}(x), t\right) u\left(\alpha_{i}(x), t\right) w_{1}\left(u\left(\alpha_{i}(x), t\right)\right)\right] d t\right] \alpha_{i}^{\prime}(x) \\
& \leq \sum_{i=1}^{n}\left[\int_{\beta_{i}\left(y_{0}\right)}^{\beta_{i}(y)}\left[f_{i}\left(\alpha_{i}(x), t\right) \varphi^{-1}\left[z\left(\alpha_{i}(x), t\right)\right] w_{1}\left(\varphi^{-1}\left[z\left(\alpha_{i}(x), t\right)\right]\right)\right] d t\right] \alpha_{i}^{\prime}(x) \\
& \leq \varphi^{-1}[z(x, y)] \sum_{i=1}^{n}\left[\int_{\beta_{i}\left(y_{0}\right)}^{\beta_{i}(y)}\left[f_{i}\left(\alpha_{i}(x), t\right) w_{1}\left(\varphi^{-1}\left[z\left(\alpha_{i}(x), t\right)\right]\right)\right] d t\right] \alpha_{i}^{\prime}(x) .
\end{aligned}
$$

Using the monotonicity of $\varphi^{-1}$ and $z$, we deduce

$$
\varphi^{-1}[z(x, y)] \geq \varphi^{-1}\left[z\left(x_{0}, y_{0}\right)\right]=\varphi^{-1}(c)>0 .
$$

From the definition of $\Omega$ and the above relation,

$$
\begin{aligned}
D_{1} \Omega(z(x, y)) & =\frac{D_{1} z(x, y)}{\varphi^{-1}[z(x, y)]} \\
& \leq \sum_{i=1}^{n}\left[\int_{\beta_{i}\left(y_{0}\right)}^{\beta_{i}(y)}\left[f_{i}\left(\alpha_{i}(x), t\right) w_{1}\left(\varphi^{-1}\left[z\left(\alpha_{i}(x), t\right)\right]\right)\right] d t\right] \alpha_{i}^{\prime}(x) .
\end{aligned}
$$


Keeping $y$ fixed in (2.10), setting $x=\sigma$ and integrating it with respect to $\sigma$ from $x_{0}$ to $x, x \in I_{1}$ and making the change of variable, we obtain

$$
\Omega(z(x, y)) \leq \Omega(c)+\sum_{i=1}^{n} I_{\alpha_{i}, \beta_{i}}\left[f_{i}(s, t) w_{1}\left(\varphi^{-1}[z(s, t)]\right)\right] .
$$

Now, an application of Lemma 2.1 to (2.11) gives

$$
z(x, y)=\Omega^{-1}\left[G^{-1}\left(G(\Omega(c))+\sum_{i=1}^{n} I_{\alpha_{i}, \beta_{i}}\left[f_{i}(s, t)\right]\right)\right] .
$$

Using (2.12) in (2.9), we get the required inequality.

If $c=0$, we carry out the above procedure with $\varepsilon>0$ instead of $c$ and subsequently let $\varepsilon \rightarrow 0$. This completes the proof.

Theorem 2.3. Let $u, f_{i}, g_{i} \in C\left(\triangle, R_{+}\right), \alpha_{i} \in C^{1}\left(I_{1}, I_{1}\right), \beta_{i} \in C^{1}\left(I_{2}, I_{2}\right)$ be nondecreasing with $\alpha_{i}(x) \leq x$ on $I_{1}, \beta_{i}(y) \leq y$ on $I_{2}$ for $i=1,2, \ldots, n$. Let $c$ be $a$ nonnegative constant. Moreover, assume that $\varphi \in C\left(R_{+}, R_{+}\right)$and $w_{1} \in C\left(R_{+}, R_{+}\right)$ are defined as in Theorem 2.2. If

$$
\varphi(u(x, y)) \leq c+\sum_{i=1}^{n} I_{\alpha_{i}, \beta_{i}}\left[f_{i}(s, t) u(s, t) w_{1}(u(s, t))+g_{i}(s, t) u(s, t)\right]
$$

for all $x \in I_{1}, y \in I_{2}$, then, for $x_{0} \leq x \leq x_{2}, y_{0} \leq y \leq y_{2}$ with $x_{2} \in I_{1}, y_{2} \in I_{2}$,

$$
u(x, y) \leq \varphi^{-1}\left\{\Omega^{-1}\left[G^{-1}\left(G[p(x, y)]+\sum_{i=1}^{n} I_{\alpha_{i}, \beta_{i}} f_{i}(s, t)\right)\right]\right\},
$$

where

$$
p(x, y)=\Omega(c)+\sum_{i=1}^{n} I_{\alpha_{i}, \beta_{i}}[g(s, t)]
$$

$\Omega(r)=\int_{r_{0}}^{r} \frac{d s}{\varphi^{-1}(s)}, \quad r \geq r_{0}>0, \quad G(z)=\int_{z_{0}}^{z} \frac{d s}{w_{1}\left[\varphi^{-1}\left(\Omega^{-1}(s)\right)\right]}, \quad z \geq z_{0}>0$,

$\Omega^{-1}, \varphi^{-1}, G^{-1}$ are, respectively, the inverses of $\Omega, \varphi, G$ and $x_{2} \in I_{1}, y_{2} \in I_{2}$ are chosen so that

$$
\begin{gathered}
G_{i}(\Omega(c))+\sum_{i=1}^{n} I_{\alpha_{i}, \beta_{i}}\left[f_{i}(s, t)+g(s, t)\right] \in \operatorname{Dom}\left(G_{i}^{-1}\right), \\
G_{i}^{-1}\left[G_{i}(\Omega(c))+\sum_{i=1}^{n} I_{\alpha_{i}, \beta_{i}}\left[f_{i}(s, t)+g(s, t)\right]\right] \in \operatorname{Dom}\left(\Omega^{-1}\right), \\
\Omega^{-1}\left\{G_{i}^{-1}\left[G_{i}(\Omega(c))+\sum_{i=1}^{n} I_{\alpha_{i}, \beta_{i}}\left[f_{i}(s, t)+g(s, t)\right]\right]\right\} \in \operatorname{Dom}\left(\varphi^{-1}\right)
\end{gathered}
$$

for all $x \in\left[x_{0}, x_{2}\right]$ and $y \in\left[y_{0}, y_{2}\right]$. 
PROOF. Let us first assume that $c>0$. Define a positive function $z(x, y)$ by

$$
z(x, y)=c+\sum_{i=1}^{n} I_{\alpha_{i}, \beta_{i}}\left[f_{i}(s, t) u(s, t) w_{1}(u(s, t))+g_{i}(s, t) u(s, t)\right] .
$$

Then $z(x, y)>0, z\left(x_{0}, y\right)=z\left(x, y_{0}\right)=c$ and (2.13) can be restated as

$$
u(x, y) \leq \varphi^{-1}[z(x, y)] .
$$

It is easy to observe that $z(x, y)$ is a continuous nondecreasing function for all $x \in I_{1}, y \in I_{2}$ and

$$
\begin{aligned}
D_{1} z(x, y)= & \sum_{i=1}^{n}\left[\int _ { \beta _ { i } ( y _ { 0 } ) } ^ { \beta _ { i } ( y ) } \left[f_{i}\left(\alpha_{i}(x), t\right) u\left(\alpha_{i}(x), t\right) w_{1}\left(u\left(\alpha_{i}(x), t\right)\right)\right.\right. \\
& \left.\left.+g_{i}\left(\alpha_{i}(x), t\right) u\left(\alpha_{i}(x), t\right)\right] d t\right] \alpha_{i}^{\prime}(x) \\
\leq & \sum_{i=1}^{n}\left[\int _ { \beta _ { i } ( y _ { 0 } ) } ^ { \beta _ { i } ( y ) } \left[f_{i}\left(\alpha_{i}(x), t\right) \varphi^{-1}\left[z\left(\alpha_{i}(x), t\right)\right] w_{1}\left(\varphi^{-1}\left[z\left(\alpha_{i}(x), t\right)\right]\right)\right.\right. \\
& \left.\left.+g_{i}\left(\alpha_{i}(x), t\right) \varphi^{-1}\left[z\left(\alpha_{i}(x), t\right)\right] d t\right] \alpha_{i}^{\prime}(x)\right] \\
\leq & \varphi^{-1}[z(x, y)] \sum_{i=1}^{n}\left[\int _ { \beta _ { i } ( y _ { 0 } ) } ^ { \beta _ { i } ( y ) } \left[f_{i}\left(\alpha_{i}(x), t\right) w_{1}\left(\varphi^{-1}\left[z\left(\alpha_{i}(x), t\right)\right]\right)\right.\right. \\
& \left.\left.+g_{i}\left(\alpha_{i}(x), t\right)\right] d t\right] \alpha_{i}^{\prime}(x) .
\end{aligned}
$$

Using the monotonicity of $\varphi^{-1}$ and $z$, we deduce

$$
\varphi^{-1}[z(x, y)] \geq \varphi^{-1}\left[z\left(x_{0}, y_{0}\right)\right]=\varphi^{-1}(c)>0 .
$$

From the definition of $\Omega$ and the above relation,

$$
\begin{aligned}
D_{1} \Omega(z(x, y))= & \frac{D_{1} z(x, y)}{\varphi^{-1}[z(x, y)]} \\
\leq & \sum_{i=1}^{n}\left[\int _ { \beta _ { i } ( y _ { 0 } ) } ^ { \beta _ { i } ( y ) } \left[f_{i}\left(\alpha_{i}(x), t\right) w_{1}\left(\varphi^{-1}\left[z\left(\alpha_{i}(x), t\right)\right]\right)\right.\right. \\
& \left.\left.+g_{i}\left(\alpha_{i}(x), t\right)\right] d t\right] \alpha_{i}^{\prime}(x) .
\end{aligned}
$$

Keeping $y$ fixed in (2.15), setting $x=\sigma$ and integrating it with respect to $\sigma$ from $x_{0}$ to $x, x \in I_{1}$ and making the change of variable, we obtain

$$
\Omega(z(x, y)) \leq \Omega(c)+\sum_{i=1}^{n} I_{\alpha_{i}, \beta_{i}}\left[f_{i}(s, t) w_{1}\left(\varphi^{-1}[z(s, t)]\right)+g_{i}(s, t)\right] .
$$


Let $x \leq X, y \leq Y$ be arbitrary numbers and denote

$$
p(x, y)=\Omega(c)+\sum_{i=1}^{n} I_{\alpha_{i}, \beta_{i}}\left[g_{i}(s, t)\right] .
$$

From the above relation, we deduce

$$
\Omega(z(x, y)) \leq p(X, Y)+\sum_{i=1}^{n} I_{\alpha_{i}, \beta_{i}}\left[f_{i}(s, t) w_{1}\left(\varphi^{-1}(z(s, t))\right)\right] .
$$

Now, an application of Lemma 2.1 gives

$$
z(x, y) \leq \Omega^{-1}\left[G^{-1}\left(G(p(X, Y))+\sum_{i=1}^{n} I_{\alpha_{i}, \beta_{i}}\left[f_{i}(s, t)\right]\right)\right] .
$$

Using the inequality (2.16) in the inequality (2.14), we get

$$
u(x, y) \leq \varphi^{-1}\left\{\Omega^{-1}\left[G^{-1}\left(G(p(X, Y))+\sum_{i=1}^{n} I_{\alpha_{i}, \beta_{i}}\left[f_{i}(s, t)\right]\right)\right]\right\} .
$$

Taking $x=X, y=Y$ in the above inequality, since $X$ and $Y$ are arbitrary, we can prove the desired inequality.

If $c=0$, we carry out the above procedure with $\varepsilon>0$ instead of $c$ and subsequently let $\varepsilon \rightarrow 0$. This completes the proof.

Corollary 2.4. Let $u, f_{i}, g_{i} \in C\left(\triangle, R_{+}\right), \alpha_{i} \in C^{1}\left(I_{1}, I_{1}\right), \beta_{i} \in C^{1}\left(I_{2}, I_{2}\right)$ be nondecreasing with $\alpha_{i}(x) \leq x$ on $I_{1}, \beta_{i}(y) \leq y$ on $I_{2}$ for $i=1,2, \ldots, n$. Let $p>1$ and $c \geq 0$ be constants. Moreover, assume that $w_{1} \in C\left(R_{+}, R_{+}\right)$is defined as in Theorem 2.2. If

$$
u^{p}(x, y) \leq c+p \sum_{i=1}^{n} I_{\alpha_{i}, \beta_{i}}\left[f_{i}(s, t) u(s, t) w_{1}(u(s, t))+g_{i}(s, t) u(s, t)\right]
$$

for all $x \in I_{1}, y \in I_{2}$, then, for $x_{0} \leq x \leq x_{2}, y_{0} \leq y \leq y_{2}$ with $x_{2} \in I_{1}, y_{2} \in I_{2}$,

$$
u(x, y) \leq\left[G^{-1}\left(G[B(x, y)]+(p-1) \sum_{i=1}^{n} I_{\alpha_{i}, \beta_{i}}\left[f_{i}(s, t)\right]\right)\right]^{1 /(p-1)},
$$

where $B(x, y)=c^{(p-1) / p}+(p-1) \sum_{i=1}^{n} I_{\alpha_{i}, \beta_{i}}\left[g_{i}(s, t)\right], G^{-1}$ is the inverse function of

$$
G(z)=\int_{z_{0}}^{z} \frac{d s}{w_{1}\left[s^{1 /(p-1)}\right]}, \quad z \geq z_{0}>0,
$$

and $x_{2} \in I_{1}, y_{2} \in I_{2}$ are chosen so that

$$
G[B(x, y)]+(p-1) \sum_{i=1}^{n} I_{\alpha_{i}, \beta_{i}}\left[f_{i}(s, t)\right] \in \operatorname{Dom}\left(G_{i}^{-1}\right)
$$

for all $x \in\left[x_{0}, x_{2}\right]$ and $y \in\left[y_{0}, y_{2}\right]$. 
TheOREM 2.5. Let $u, f_{i}, g_{i} \in C\left(\triangle, R_{+}\right), \alpha_{i} \in C^{1}\left(I_{1}, I_{1}\right), \beta_{i} \in C^{1}\left(I_{2}, I_{2}\right)$ be nondecreasing with $\alpha_{i}(x) \leq x$ on $I_{1}, \beta_{i}(y) \leq y$ on $I_{2}$ for $i=1,2, \ldots, n$. Let $\varphi \in$ $C\left(R_{+}, R_{+}\right)$be an increasing function with $\varphi(\infty)=\infty$ and $c$ be a nonnegative constant. Moreover, let $w_{1}, w_{2} \in C\left(R_{+}, R_{+}\right)$be nondecreasing functions with $w_{1}, w_{2}>0$ on $(0, \infty)$. If

$$
\varphi(u(x, y)) \leq c+\sum_{i=1}^{n} I_{\alpha_{i}, \beta_{i}}\left[f_{i}(s, t) w_{1}(u(s, t))+g_{i}(s, t) w_{2}(u(s, t))\right]
$$

for all $x \in I_{1}, y \in I_{2}$, then, for $x_{0} \leq x \leq x_{2}, y_{0} \leq y \leq y_{2}$ with $x_{2} \in I_{1}, y_{2} \in I_{2}$, we have the following property.

(1) For the case $w_{2}(u) \leq w_{1}(u)$,

$$
u(x, y) \leq \varphi^{-1}\left\{G_{1}^{-1}\left(G_{1}(c)+\sum_{i=1}^{n} I_{\alpha_{i}, \beta_{i}}\left[f_{i}(s, t)+g(s, t)\right]\right)\right\} .
$$

(2) For the case $w_{1}(u) \leq w_{2}(u)$,

$$
u(x, y) \leq \varphi^{-1}\left\{G_{2}^{-1}\left(G_{2}(c)+\sum_{i=1}^{n} I_{\alpha_{i}, \beta_{i}}\left[f_{i}(s, t)+g(s, t)\right]\right)\right\},
$$

where

$$
G_{i}(r)=\int_{r_{0}}^{z} \frac{d s}{w_{i}\left[\varphi^{-1}(s)\right]}, \quad r \geq r_{0}>0 \quad(i=1,2),
$$

and $\varphi^{-1}, G_{i}^{-1}$ are, respectively, the inverses of $\varphi, G_{i}$ and $x_{2} \in I_{1}, y_{2} \in I_{2}$ are chosen so that

$$
G_{i}(c)+\sum_{i=1}^{n} I_{\alpha_{i}, \beta_{i}}\left[f_{i}(s, t)+g_{i}(c, t)\right] \in \operatorname{Dom}\left(G_{i}^{-1}\right)
$$

for all $x \in\left[x_{0}, x_{2}\right]$ and $y \in\left[y_{0}, y_{2}\right]$.

PROOF. Let us first assume that $c>0$. Define a positive function $z(x, y)$ by

$$
z(x, y)=c+\sum_{i=1}^{n} I_{\alpha_{i}, \beta_{i}}\left[f_{i}(s, t) w_{1}(u(s, t))+g_{i}(s, t) w_{2}(u(s, t))\right] .
$$

Then $z(x, y)>0, z\left(x_{0}, y\right)=z\left(x, y_{0}\right)=c$ and (2.17) can be restated as

$$
u(x, y) \leq \varphi^{-1}[z(x, y)] .
$$

It is easy to observe that $z(x, y)$ is a continuous nondecreasing function for all $x \in I_{1}, y \in I_{2}$ and

$$
\begin{aligned}
D_{1} z(x, y) \leq & \sum_{i=1}^{n}\left[\int _ { \beta _ { i } ( y _ { 0 } ) } ^ { \beta _ { i } ( y ) } \left[f_{i}\left(\alpha_{i}(x), t\right) w_{1}\left(\varphi^{-1}\left[z\left(\alpha_{i}(x), t\right)\right]\right)\right.\right. \\
& \left.\left.+g_{i}\left(\alpha_{i}(x), t\right) w_{2}\left(\varphi^{-1}\left[z\left(\alpha_{i}(x), t\right)\right]\right)\right] d t\right] \alpha_{i}^{\prime}(x) .
\end{aligned}
$$


(1) When $w_{2}(u) \leq w_{1}(u)$, using the monotonicity of $\varphi^{-1}$ and $z$, we deduce

$$
D_{1} z(x, y) \leq w_{1}\left(\varphi^{-1}[z(x, y)]\right) \sum_{i=1}^{n}\left[\int_{\beta_{i}\left(y_{0}\right)}^{\beta_{i}(y)}\left[f_{i}\left(\alpha_{i}(x), t\right)+g_{i}\left(\alpha_{i}(x), t\right)\right] d t\right] \alpha_{i}^{\prime}(x) .
$$

From the definition of $G_{1}$ and the above relation,

$$
D_{1} G_{1}(z(x, y)) \leq \sum_{i=1}^{n}\left[\int_{\beta_{i}\left(y_{0}\right)}^{\beta_{i}(y)}\left[f_{i}\left(\alpha_{i}(x), t\right)+g_{i}\left(\alpha_{i}(x), t\right)\right] d t\right] \alpha_{i}^{\prime}(x) .
$$

Keeping $y$ fixed in (2.19), setting $x=\sigma$ and integrating it with respect to $\sigma$ from $x_{0}$ to $x, x \in I_{1}$ and making the change of variable, we obtain

$$
z(x, y) \leq G_{1}^{-1}\left(G(c)+\sum_{i=1}^{n} I_{\alpha_{i}, \beta_{i}}\left[f_{i}(s, t)+g_{i}(s, t)\right]\right)
$$

Using (2.18) and (2.20) in (2.17), we get the required inequality.

If $c=0$, we carry aut the above procedure with $\varepsilon>0$ instead of $c$ and subsequently let $\varepsilon \rightarrow 0$.

(2) When $w_{1}(u) \leq w_{2}(u)$, the proof can be completed similarly. This completes the proof.

THEOREM 2.6. Let $u, f_{i}, g_{i} \in C\left(\triangle, R_{+}\right), \alpha_{i} \in C^{1}\left(I_{1}, I_{1}\right), \beta_{i} \in C^{1}\left(I_{2}, I_{2}\right)$ be nondecreasing with $\alpha_{i}(x) \leq x$ on $I_{1}, \beta_{i}(y) \leq y$ on $I_{2}$ for $i=1,2, \ldots, n$. Let $\varphi \in$ $C\left(R_{+}, R_{+}\right)$be an increasing function with $\varphi(\infty)=\infty$ and $c$ be a nonnegative constant. Moreover, let $w_{1}, w_{2} \in C\left(R_{+}, R_{+}\right)$be a nondecreasing function with $w_{1}, w_{2}>0$ on $(0, \infty)$. If

$$
\varphi(u(x, y)) \leq c+\sum_{i=1}^{n} I_{\alpha_{i}, \beta_{i}}\left[f_{i}(s, t) u(s, t) w_{1}(u(s, t))+g_{i}(s, t) u(s, t) w_{2}(u(s, t))\right]
$$

for all $x \in I_{1}, y \in I_{2}$, then, for $x_{0} \leq x \leq x_{2}, y_{0} \leq y \leq y_{2}$ with $x_{2} \in I_{1}, y_{2} \in I_{2}$, we have the following property.

(1) For the case $w_{2}(u) \leq w_{1}(u)$,

$$
u(x, y) \leq \varphi^{-1}\left\{\Omega^{-1}\left[H_{1}^{-1}\left(H_{1}(\Omega(c))+\sum_{i=1}^{n} I_{\alpha_{i}, \beta_{i}}\left[f_{i}(s, t)+g_{i}(s, t)\right]\right)\right]\right\} .
$$

(2) For the case $w_{1}(u) \leq w_{2}(u)$,

$$
u(x, y) \leq \varphi^{-1}\left\{\Omega^{-1}\left[H_{2}^{-1}\left(H_{2}(\Omega(c))+\sum_{i=1}^{n} I_{\alpha_{i}, \beta_{i}}\left[f_{i}(s, t)+g_{i}(s, t)\right]\right)\right]\right\}
$$


where

$$
\begin{gathered}
\Omega(r)=\int_{r_{0}}^{r} \frac{d s}{\varphi^{-1}(z(s))}, \quad r \geq r_{0}>0, \\
H_{i}(z)=\int_{z_{0}}^{z} \frac{d s}{w_{i}\left[\varphi^{-1}\left(\Omega^{-1}(s)\right)\right]}, \quad z \geq z_{0}>0 \quad(i=1,2),
\end{gathered}
$$

$\varphi^{-1}, \Omega^{-1}, H_{i}^{-1}$ are, respectively, the inverses of $\varphi, \Omega, H_{i}$ for $i=1,2$ and $x_{2} \in I_{1}$, $y_{2} \in I_{2}$ are chosen so that

$$
\begin{gathered}
H_{i}(\Omega(c))+\sum_{i=1}^{n} I_{\alpha_{i}, \beta_{i}}\left[f_{i}(s, t)+g_{i}(s, t)\right] \in \operatorname{Dom}\left(H_{i}^{-1}\right), \\
H_{i}^{-1}\left(H_{i}(\Omega(c))+\sum_{i=1}^{n} I_{\alpha_{i}, \beta_{i}}\left[f_{i}(s, t)+g_{i}(s, t)\right]\right) \in \operatorname{Dom}\left(\Omega^{-1}\right)
\end{gathered}
$$

for all $x \in\left[x_{0}, x_{2}\right]$ and $y \in\left[y_{0}, y_{2}\right]$.

PROOF. Let us first assume that $c>0$. Define a positive function $z(x, y)$ by

$$
z(x, y)=c+\sum_{i=1}^{n} I_{\alpha_{i}, \beta_{i}}\left[f_{i}(s, t) u(s, t) w_{1}(u(s, t))+g_{i}(s, t) u(s, t) w_{2}(u(s, t))\right] .
$$

Then $z(x, y)>0, z\left(x_{0}, y\right)=z\left(x, y_{0}\right)=c$ and (2.21) can be restated as

$$
u(x, y) \leq \varphi^{-1}[z(x, y)] .
$$

It is easy to observe that $z(x, y)$ is a continuous nondecreasing function for all $x \in I_{1}$, $y \in I_{2}$ and

$$
\begin{aligned}
D_{1} z(x, y) \leq & \varphi^{-1}[z(x, y)] \sum_{i=1}^{n}\left[\int _ { \beta _ { i } ( y _ { 0 } ) } ^ { \beta _ { i } ( y ) } \left[f_{i}\left(\alpha_{i}(x), t\right) w_{1}\left(\varphi^{-1}\left[z\left(\alpha_{i}(x), t\right)\right]\right)\right.\right. \\
& \left.\left.+g_{i}\left(\alpha_{i}(x), t\right) w_{2}\left(\varphi^{-1}\left[z\left(\alpha_{i}(x), t\right)\right]\right)\right] d t\right] \alpha_{i}^{\prime}(x) .
\end{aligned}
$$

Using the monotonicity of $\varphi^{-1}$ and $z$, we deduce

$$
\varphi^{-1}[z(x, y)] \geq \varphi^{-1}\left[z\left(x_{0}, y_{0}\right)\right]=\varphi^{-1}(c)>0 .
$$

From the definition of $\Omega$ and the above relation,

$$
\begin{aligned}
D_{1} \Omega(z(x, y)) \leq & \sum_{i=1}^{n}\left[\int _ { \beta _ { i } ( y _ { 0 } ) } ^ { \beta _ { i } ( y ) } \left[f_{i}\left(\alpha_{i}(x), t\right) w_{1}\left(\varphi^{-1}\left[z\left(\alpha_{i}(x), t\right)\right]\right)\right.\right. \\
& \left.\left.+g_{i}\left(\alpha_{i}(x), t\right) w_{2}\left(\varphi^{-1}\left[z\left(\alpha_{i}(x), t\right)\right]\right)\right] d t\right] \alpha_{i}^{\prime}(x) .
\end{aligned}
$$


Keeping $y$ fixed in (2.21), setting $x=\sigma$ and integrating it with respect to $\sigma$ from $x_{0}$ to $x, x \in I_{1}$ and making the change of variable, we obtain

$\Omega(z(x, y)) \leq \Omega(c)+\sum_{i=1}^{n} I_{\alpha_{i}, \beta_{i}}\left[f_{i}(s, t) w_{1}\left(\varphi^{-1}[z(s, t)]\right)+g_{i}(s, t) w_{2}\left(\varphi^{-1}[z(s, t)]\right)\right]$.

Now, an application of Theorem 2.5 to (2.23), we can prove the desired inequalities.

If $c=0$, we carry out the above procedure with $\varepsilon>0$ instead of $c$ and subsequently let $\varepsilon \rightarrow 0$. This completes the proof.

\section{Applications}

In this section, we will show that our results are useful in proving the global existence of solutions to certain differential equations with time delay. First consider the partial differential equation involving several retarded arguments with the initial boundary conditions

$$
\begin{aligned}
& D_{2}\left(z^{p-1}(x, y) D_{1} z(x, y)\right) \\
& \quad=F\left[x, y, z\left(x-h_{1}(x), y-k_{1}(y)\right), \ldots, z\left(x-h_{n}(x), y-k_{n}(y)\right)\right], \\
& z\left(x, y_{0}\right)=e_{1}(x), \quad z\left(x_{0}, y\right)=e_{2}(y), \quad e_{1}\left(x_{0}\right)=e_{2}\left(y_{0}\right)=0
\end{aligned}
$$

where $p>1$ is a constant, $F \in C\left(\triangle \times R^{n}, R\right), e_{1} \in C^{1}\left(I_{1}, R_{+}\right), e_{2} \in C^{1}\left(I_{2}, R_{+}\right)$ and $h_{i} \in C^{1}\left(I_{1}, R_{+}\right), k_{i} \in C^{1}\left(I_{2}, R_{+}\right)$are nonincreasing and such that $x-h_{i}(x) \geq 0$, $x-h_{i}(x) \in C^{1}\left(I_{1}, I_{1}\right), y-k_{i}(y) \geq 0, y-k_{i}(y) \in C^{1}\left(I_{2}, I_{2}\right), h_{i}^{\prime}(x)<1, k_{i}^{\prime}(y)<1$ and $h_{i}\left(x_{0}\right)=k_{i}\left(y_{0}\right)=0$ for $i=1, \ldots, n$ and $x \in I_{1}, y \in I_{2}$.

The following theorem deals with a boundedness on the solution of (3.1).

THEOREM 3.1. Assume that $F: \Delta \times R^{n} \rightarrow R$ is a continuous function for which there exists continuous nonnegative functions $f_{i}(x, y), g_{i}(x, y)$ for $i=1, \ldots, n$ and $x \in I_{1}, y \in I_{2}$ such that

$$
\left\{\begin{array}{l}
\left|F\left(x, y, u_{1}, \ldots, u_{n}\right)\right| \leq \sum_{i=1}^{n}\left\{f_{i}(x, y)\left|u_{i}\right| w_{1}\left(\left|u_{i}\right|\right)+g_{i}(x, y)\left|u_{i}\right|\right\} \\
\left|e_{1}(x)+e_{2}(y)\right| \leq c
\end{array}\right.
$$

where $c$ is a constant. Let

$$
M_{i}=\max _{x \in I_{1}} \frac{1}{1-h_{i}^{\prime}(x)}, \quad N_{i}=\max _{y \in I_{2}} \frac{1}{1-k_{i}^{\prime}(y)}, \quad i=1, \ldots, n .
$$

If $z(x, y)$ is any solution of the problem (3.1) with the condition (3.2), then

$$
\|z(x, y)\| \leq\left[G^{-1}\left(G[\bar{B}(x, y)]+(p-1) \sum_{i=1}^{n} I_{\phi_{i}, \psi_{i}}\left[\overline{f_{i}}(s, t)\right]\right)\right]^{1 /(p-1)},
$$


where $\bar{B}(x, y)=c^{(p-1) / p}+(p-1) \sum_{i=1}^{n} I_{\alpha_{i}, \beta_{i}}\left[\overline{g_{i}}(s, t)\right], G^{-1}$ is the inverse function of

$$
G(z)=\int_{z_{0}}^{z} \frac{d s}{w_{1}\left[s^{1 /(p-1)}\right]}, \quad z \geq z_{0}>0,
$$

for all $(x, y) \in \triangle_{1}$, where $\phi(x)=x-h_{i}(x), \psi(y)=y-k_{i}(y)$ and

$$
\begin{aligned}
& \overline{f_{i}}(\sigma, \tau)=M_{i} N_{i} f_{i}\left(\sigma+h_{i}(s), \tau+k_{i}(t)\right), \\
& \overline{g_{i}}(\sigma, \tau)=M_{i} N_{i} g_{i}\left(\sigma+h_{i}(s), \tau+k_{i}(t)\right), \quad \sigma, s \in I_{1}, \quad \tau, t \in I_{2} .
\end{aligned}
$$

PROOF. It is easy to see that the solution $z(x, y)$ of the problem (3.1) satisfies the equivalent integral equation

$$
\begin{aligned}
z^{p}(x, y)= & \int_{x_{0}}^{x} \int_{y_{0}}^{y} F\left(s, t, z\left(s-h_{1}(s), t-k_{1}(t)\right), \ldots, z\left(s-h_{n}(s), t-k_{n}(t)\right)\right) d t d s \\
& +e_{1}(x)+e_{2}(y) .
\end{aligned}
$$

From (3.3) and making the change of variables, we have

$$
\begin{aligned}
|z(x, y)|^{p} & \leq c+p I_{x, y} \sum_{i=1}^{n}\left\{f_{i}(x, y)\left|z\left(s-h_{i}(s), t-k_{i}(t)\right)\right| w_{1}\left(\left|z\left(s-h_{i}(s), t-k_{i}(t)\right)\right|\right)\right. \\
& \left.+g_{i}(x, y)\left|z\left(s-h_{i}(s), t-k_{i}(t)\right)\right|\right\} \\
\leq & c+p \sum_{i=1}^{n} I_{\phi_{i}, \psi_{i}}\left\{\overline{f_{i}}(\sigma, \tau)|z(\sigma, \tau)| w_{1}(|z(\sigma, \tau)|)+\overline{g_{i}}(x, y)|z(\sigma, \tau)|\right\} . \quad \text { (3.5) }
\end{aligned}
$$

Now, a suitable application of the inequality given in Corollary 2.4 to (3.5) yields the desired result. This completes the proof.

REMARK 1. Consider the partial differential equation (3.1) with the initial boundary condition (3.2). Assume that $F: \triangle \times R^{n} \rightarrow R$ is a continuous function for which there exists continuous nonnegative functions $g_{i}(x, y)$ such that

$$
\left|F\left(x, y, u_{1}, \ldots, u_{n}\right)\right| \leq \sum_{i=1}^{n} g_{i}(x, y)\left|u_{i}\right| .
$$

Let $M_{i}$ and $N_{i}$ be functions defined by (3.4). If $z(x, y)$ is any solution of the problem (3.1) with the condition (3.2), then the solution $z(x, y)$ can be written as

$$
\begin{aligned}
z^{p}(x, y)= & \int_{x_{0}}^{x} \int_{y_{0}}^{y} F\left(s, t, z\left(s-h_{1}(s), t-k_{1}(t)\right), z\left(s-h_{n}(s), t-k_{n}(t)\right)\right) d t d s \\
& +e_{1}(x)+e_{2}(y) .
\end{aligned}
$$


From (3.6) and (3.7), making the change of variables, we have

$$
\begin{aligned}
|z(x, y)|^{p} & \leq c+p \int_{x_{0}}^{x} \int_{y_{0}}^{y} \sum_{i=1}^{n} g_{i}(x, y)\left|z\left(s-h_{i}(s), t-k_{i}(t)\right)\right| d t d s \\
& \leq c+p \sum_{i=1}^{n} I_{\phi_{i}, \psi_{i}}\left[\overline{g_{i}}(x, y)|z(\sigma, \tau)|\right] .
\end{aligned}
$$

Now, a suitable application of the inequality given in Corollary 2.4 to (3.8) yields

$$
|z(x, y)| \leq\left[c^{(p-1) / p}+(p-1) \sum_{i=1}^{n} I_{\alpha_{i}, \beta_{i}}\left[\overline{g_{i}}(s, t)\right]\right]^{1 /(p-1)}
$$

for all $(x, y) \in \triangle$, where $\phi(x)=x-h_{i}(x), \psi(y)=y-k_{i}(y)$ and

$$
\overline{g_{i}}(\sigma, \tau)=M_{i} N_{i} g_{i}\left(\sigma+h_{i}(s), \tau+k_{i}(t)\right), \quad \sigma, s \in I_{1}, \quad \tau, t \in I_{2}
$$

In the following, we present an application of the inequality given in Section 2 to study the boundedness of the solutions of the initial boundary value problem for the hyperbolic partial delay differential equations of the form

$$
\begin{aligned}
& D_{2}\left(D_{1} \varphi(z(x, y))\right) \\
& \quad=F\left[x, y, z\left(x-h_{1}(x), y-k_{1}(y)\right), \ldots, z\left(x-h_{n}(x), y-k_{n}(y)\right)\right], \\
& \varphi\left(z\left(x, y_{0}\right)\right)=e_{3}(x), \quad \varphi\left(z\left(x_{0}, y\right)\right)=e_{4}(y), \quad e_{3}\left(x_{0}\right)=e_{4}\left(y_{0}\right)=0,
\end{aligned}
$$

where $\varphi \in C\left(R_{+}, R_{+}\right)$is an increasing function with $\varphi(0)=0, \varphi(\infty)=\infty, F \in$ $C\left(\triangle \times R^{n}, R\right), e_{3} \in C^{1}\left(I_{1}, R_{+}\right), e_{4} \in C^{1}\left(I_{2}, R_{+}\right)$and $h_{i} \in C^{1}\left(I_{1}, R_{+}\right), k_{i} \in C^{1}\left(I_{2}, R_{+}\right)$ are nonincreasing and such that

$$
\begin{gathered}
x-h_{i}(x) \geq 0, \quad x-h_{i}(x) \in C^{1}\left(I_{1}, I_{1}\right), \\
y-k_{i}(y) \geq 0, \quad y-k_{i}(y) \in C^{1}\left(I_{2}, I_{2}\right), \\
h_{i}^{\prime}(x)<1, \quad k_{i}^{\prime}(y)<1, \quad h_{i}\left(x_{0}\right)=k_{i}\left(y_{0}\right)=0
\end{gathered}
$$

for all $i=1, \ldots, n$ and $x \in I_{1}, y \in I_{2}$.

The following theorem deals with a boundedness on the solution of (3.9).

THEOREM 3.2. Assume that $F: \triangle \times R^{n} \rightarrow R$ is a continuous function for which there exists continuous nonnegative functions $f_{i}(x, y), g_{i}(x, y)$ for all $i=1, \ldots, n$ and $x \in I_{1}, y \in I_{2}$ such that

$$
\left\{\begin{array}{l}
\left|F\left(x, y, u_{1}, \ldots, u_{n}\right)\right| \leq \sum_{i=1}^{n}\left\{f_{i}(x, y)\left|u_{i}\right| w_{1}\left(\left|u_{i}\right|\right)+g_{i}(x, y)\left|u_{i}\right| w_{2}\left(\left|u_{i}\right|\right)\right\} \\
\left|e_{3}(x)+e_{4}(y)\right| \leq c
\end{array}\right.
$$


where $c$ is a constant. Let

$$
M_{i}=\max _{x \in I_{1}} \frac{1}{1-h_{i}^{\prime}(x)}, \quad N_{i}=\max _{y \in I_{2}} \frac{1}{1-k_{i}^{\prime}(y)}, \quad i=1, \ldots, n .
$$

If $z(x, y)$ is any solution of the problem (3.9) with the condition (3.10), then, for the case $w_{2}(u) \leq w_{1}(u)$,

$$
|z(x, y)| \leq \varphi^{-1}\left\{\Omega^{-1}\left[H_{1}^{-1}\left(H_{1}[\Omega(c)]+\sum_{i=1}^{n} I_{\phi_{i}, \psi_{i}}\left[\overline{f_{i}}(\sigma, \tau)+\overline{g_{i}}(\sigma, \tau)\right]\right)\right]\right\}
$$

where $\varphi^{-1}, \Omega^{-1}, H_{1}^{-1}$ are, respectively, inverse functions of $\varphi, \Omega, H_{1}$ for all $(x, y)$ $\in \triangle, \Omega(r), H_{1}(z)$ are as in Theorem 2.6, $\phi(x)=x-h_{i}(x), \psi(y)=y-k_{i}(y)$ and

$$
\begin{gathered}
\overline{f_{i}}(\sigma, \tau)=M_{i} N_{i} f_{i}\left(\sigma+h_{i}(s), \tau+k_{i}(t)\right), \\
\overline{g_{i}}(\sigma, \tau)=M_{i} N_{i} g_{i}\left(\sigma+h_{i}(s), \tau+k_{i}(t)\right)
\end{gathered}
$$

for all $\sigma, s \in I_{1}$ and $\tau, t \in I_{2}$.

PROOF. It is easy to see that the solution $z(x, y)$ of the problem (3.9) satisfies the equivalent integral equation:

$$
\begin{aligned}
& \varphi(z(x, y)) \\
& \qquad \begin{array}{l}
\int_{x_{0}}^{x} \int_{y_{0}}^{y} F\left(s, t, z\left(s-h_{1}(s), t-k_{1}(t)\right), \ldots, z\left(s-h_{n}(s), t-k_{n}(t)\right)\right) d t d s \\
\quad+e_{3}(x)+e_{4}(y) .
\end{array}
\end{aligned}
$$

From (3.11) and making the change of variables, we have

$$
\begin{aligned}
\varphi(|z(x, y)|) \leq & c+\sum_{i=1}^{n} I_{x, y}\left[f_{i}(x, y)\left|z\left(s-h_{i}(s), t-k_{i}(t)\right)\right|\right. \\
& \times w_{1}\left(\left|z\left(s-h_{i}(s), t-k_{i}(t)\right)\right|\right) \\
& \left.+g_{i}(x, y)\left|z\left(s-h_{i}(s), t-k_{i}(t)\right)\right| w_{2}\left(\left|z\left(s-h_{i}(s), t-k_{i}(t)\right)\right|\right)\right] \\
\leq & c+\sum_{i=1}^{n} I_{\phi_{i}, \psi_{i}}\left\{\overline{f_{i}}(\sigma, \tau)|z(\sigma, \tau)| w_{1}(|z(\sigma, \tau)|)\right. \\
& \left.+\overline{g_{i}}(x, y)|z(\sigma, \tau)| w_{2}(|z(\sigma, \tau)|)\right\} .
\end{aligned}
$$

Now, a suitable application of the inequality given in Theorem 2.6 (1) to (3.13) yields the desired result. This completes the proof.

REMARK 2. Consider the partial differential equation (3.9) with the initial boundary condition (3.10). Assume that $F: \triangle \times R^{n} \rightarrow R$ is a continuous function for which 
there exists continuous nonnegative functions $g_{i}(x, y)$ such that

$$
\left|F\left(x, y, u_{1}, \ldots, u_{n}\right)\right| \leq \sum_{i=1}^{n} g_{i}(x, y)\left|u_{i}\right| .
$$

Let $M_{i}$ and $N_{i}$ be functions defined by (3.12). If $z(x, y)$ is any solution of the problem (3.9) with the condition (3.10), then the solution $z(x, y)$ can be written as

$$
\begin{aligned}
& \varphi(z(x, y)) \\
& \quad=\int_{x_{0}}^{x} \int_{y_{0}}^{y} F\left(s, t, z\left(s-h_{1}(s), t-k_{1}(t)\right), \ldots, z\left(s-h_{n}(s), t-k_{n}(t)\right)\right) d t d s \\
& \quad+e_{3}(x)+e_{4}(y) .
\end{aligned}
$$

From (3.14) and (3.15) making the change of variables, we have

$$
\begin{aligned}
\varphi(|z(x, y)|) & \leq c+\int_{x_{0}}^{x} \int_{y_{0}}^{y} \sum_{i=1}^{n} g_{i}(x, y)\left|z\left(s-h_{i}(s), t-k_{i}(t)\right)\right| d t d s \\
& \leq c+\sum_{i=1}^{n} I_{\phi_{i}, \psi_{i}}\left[\overline{g_{i}}(x, y)|z(\sigma, \tau)|\right] .
\end{aligned}
$$

Now, a suitable application of the inequality given in Theorem 2.3 to (3.16) yields

$$
|z(x, y)| \leq \varphi^{-1}\left\{\Omega^{-1}\left(\Omega(c)+\sum_{i=1}^{n} I_{\phi_{i}, \psi_{i}}\left[\overline{g_{i}}(\sigma, \tau)\right]\right)\right\}
$$

for all $(x, y) \in \triangle$, where $\phi(x)=x-h_{i}(x), \psi(y)=y-k_{i}(y)$ and

$$
\overline{g_{i}}(\sigma, \tau)=M_{i} N_{i} g_{i}\left(\sigma+h_{i}(s), \tau+k_{i}(t)\right), \quad \sigma, s \in I_{1}, \tau, t \in I_{2} .
$$

\section{Acknowledgements}

This paper was supported by the Korea Research Foundation Grant funded by the Korean Government (MOEHRD) (KRF-2007-313-C00040).

\section{References}

[1] D. Bainov and P. Simeonov, Integral inequalities and applications (Kluwer Academic Publishers, Dordrecht, 1992).

[2] W. S. Cheung, "Some new nonlinear inequalities and applications to boundary value problems", Nonlinear Anal. 64 (2006) 2112-2128.

[3] W. S. Cheung and Q. H. Ma, "On certain new Gronwall-Ou-Iang type integral inequalities in two variables and their applications", J. Inequal. Appl. 4 (2005) 347-361.

[4] Y. J. Cho, S. S. Dragomir and Y.-H. Kim, "On some integral inqualities with iterated integrals", J. Korean Math. Soc. 43 (2006) 563-578.

[5] S. S. Dragomir and Y.-H. Kim, "On certain new integral inequalities and their applications", J. Inequal. Pure Appl. Math. 3 (2002) Article 65, ONLINE http://jipam.vu.edu.au/. 
[6] S. S. Dragomir and Y.-H. Kim, "Some integral inequalities for function of two variables", Electron. J. Differential Equations 10 (2003) 1-13.

[7] O. Lipovan, "A retarded integral inequality and its applications", J. Math. Anal. Appl. 285 (2003) 336-443.

[8] Q.-H. Ma and J. Pečarić, "Some new nonlinear retarded integral inequalities and their applications", Math. Inequal. Appl. 9 (2006) 617-632.

[9] L. Ou-Iang, "The boundedness of solutions of linear differential equations $y^{\prime \prime}+A(t) y=0$ ", Shuxue Jinzhan 3 (1957) 409-415.

[10] B. G. Pachpatte, "On some new inequalities related to certain inequalities in the theory of differential equations", J. Math. Anal. Appl. 189 (1995) 128-144.

[11] B. G. Pachpatte, Inequalities for differential and integral equations (Academic Press, New York, 1998).

[12] B. G. Pachpatte, "Explicit bounds on certain integral inequalities", J. Math. Anal. Appl. 267 (2002) 48-61.

[13] B. G. Pachpatte, "On some new nonlinear retarded integral inequalities", J. Inequal. Pure Appl. Math. 5 (2004) Article 80, ONLINE http://jipam.vu.edu.au/. 